\title{
High-valent states in cobalt and nickel oxygen-evolving catalysts and their role in O-O bond formation
}

\author{
Ryan G. Hadt ${ }^{*}, \dagger$ \\ ${ }^{\dagger}$ Chemical Sciences and Engineering Division, Argonne National Laboratory, Lemont, \\ Illinois 60439, United States
}

The solar-to-fuels conversion is a promising alternative to traditional energy sources such as fossil fuels, and the overall efficiency relies heavily on catalysts of the oxygen evolution half-reaction (i.e., $2 \mathrm{H}_{2} \mathrm{O} \rightarrow \mathrm{O}_{2}+4 \mathrm{H}^{+}+4 \mathrm{e}^{-}$). This four-electron, four-proton coupled reaction ultimately provides the reducing equivalents for solar fuels synthesis. Earth-abundant first-row transition metal oxides of cobalt, nickel, and their mixed-metal forms can drive this half-reaction at relatively low overpotentials. This presentation will discuss recent studies utilizing a combination of in situ and ex situ optical and X-ray spectroscopies, including resonant inelastic X-ray scattering, on oxygen-evolving thin films and their molecular and heterogeneous inorganic analogs. Experiment coupled with density functional theory calculations has provided insights into the electronic structures of the high-valent states involved in the mechanism of $\mathrm{O}-\mathrm{O}$ bond formation. 